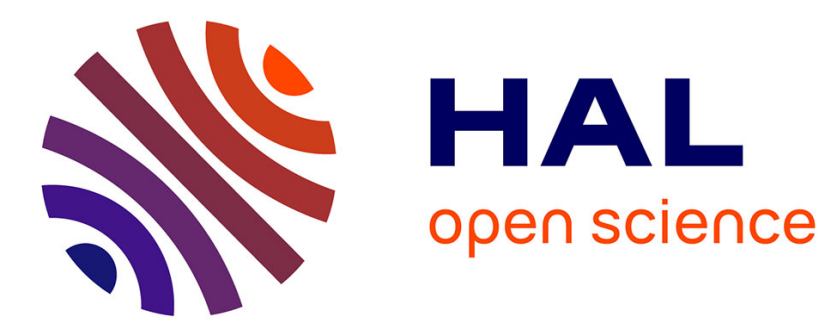

\title{
Réflexions sur les outils de mise en ouvre des recommandations alimentaires. Application aux vaches laitières dans les conditions françaises
}

J.B. Coulon, Jacques Agabriel

\section{To cite this version:}

J.B. Coulon, Jacques Agabriel. Réflexions sur les outils de mise en œuvre des recommandations alimentaires. Application aux vaches laitières dans les conditions françaises. Productions Animales, 1992, 5 (5), pp.347-353. hal-00895990

\section{HAL Id: hal-00895990 \\ https://hal.science/hal-00895990}

Submitted on 1 Jan 1992

HAL is a multi-disciplinary open access archive for the deposit and dissemination of scientific research documents, whether they are published or not. The documents may come from teaching and research institutions in France or abroad, or from public or private research centers.
L'archive ouverte pluridisciplinaire HAL, est destinée au dépôt et à la diffusion de documents scientifiques de niveau recherche, publiés ou non, émanant des établissements d'enseignement et de recherche français ou étrangers, des laboratoires publics ou privés. 
INRA Prod. Anim.,

1992, 5 (5), 347-353.

\section{J.B. COULON et J. AGABRIEL}

INRA Laboratoire Adaptation des Herbivores aux Milieux,

Theix, 63122 St-Genès Champanelle

\section{Réflexions sur les outils de mise en cuvre des recommandations alimentaires. Application aux vaches laitières dans les conditions françaises.}

L'amélioration des connaissances concernant les grandes fonctions biologiques (ingestion, digestion, métabolisme...) et les synthèses réalisées à l'échelle de l'animal au cours des dernières années en France comme à l'étranger (INRA 1988, NRC 1988, AFRC 1990, CSIRO 1990) ont conduit à des modifications plus ou moins importantes des systèmes d'alimentation des ruminants. Ces modifications se sont accompagnées d'une complexité croissante à la fois des modèles de calcul des besoins des animaux et de ceux permettant le calcul des apports alimentaires (capacité d'ingestion par exemple). Pour déterminer la meilleure ration possible dans les systèmes d'alimentation français, des outils spécifiques d'aide au calcul ont été élaborés. Ils permettent par exemple l'analyse des conséquences de modifications légères des apports alimentaires sur les performances, ou celle de l'application, à l'échelle d'un troupeau, d'une ration donnée, calculée pour un animal moyen. La démarche de rationnement a aussi été modifiée, pour être applicable dans tous les cas de figures, indépendamment des conditions d'utilisation pratiques, et de manière à pouvoir intégrer les améliorations ultérieures, et à être facilement utilisée sous forme informatique. Pour les vaches laitières, on est ainsi passé de recommandations pratiques moyennes intégrant l'ensemble des connaissances et de l'expertise, qui se traduisaient par des règles simplifiées facilement utilisables sur le terrain (lait permis par la ration de base, rythme de distribution des concentrés, densité énergétique d'une ration complète...), à des normes biologiques visant à répondre à un objectif donné, associées à des outils d'analyse des variations attendues autour de cet optimum. Les règles pratiques d'utilisation (variables suivant les situations) ne sont alors qu'un résultat d'une démarche systématique (et applicable pour toutes les catégories de ruminants). On est ainsi capable de mieux définir les limites d'application de ces règles pratiques.

\section{Résumé}

\begin{abstract}
Les systèmes d'alimentation des ruminants et les recomandations alimentaires qui en découlent ont fait l'objet de modifications sensibles au cours de ces dernières années, en France comme à l'étranger. Parallèlement, les outils d'application de ces recommandations se sont diversifiés. En France, la plupart de ces outils se réfèrent aux recommandations de l'INRA. Il est donc important de bien différencier les recommandations alimentaires des outils qui permettent de les mettre en oeuvre. Parmi ces outils, on peut distinguer les tables et tableaux (qui fournissent des valeurs d'aliment ou de besoins, bases de calculs ultérieurs), les ouvrages présentant sous forme simplifiée et précalculée des solutions pratiques directement utilisables sur le terrain, et les logiciels informatiques parmi lesquels le logiciel INRAtion. Dans cette étude, les différentes caractéristiques d'un outil de mise en oeuvre des recommandations alimentaires sont illustrées à partir d'un exemple de rationnement chez la vache laitière. L'intérêt et les développements envisagés des outils informatiques sont discutés.
\end{abstract}

Le développement de la micro-informatique a conduit tout naturellement à l'intégration de modules de rationnement dans des logiciels plus généraux de conduite des troupeaux, ou à la création de logiciels de rationnement spécifiques. Mais les outils informatiques, à coté des nombreux avantages qu'ils peuvent présenter, ont aussi certaines limites et des contraintes (coût, maniabilité et, surtout, risque de perte de réflexion et de sens critique de l'utilisateur).

L'objectif de cette présentation n'est pas de comparer ces différents outils (dont la liste serait de toute façon incomplète, et dont la plupart des étrangers ne sont pas disponibles 
ou utilisables en France) de manière exhaustive, mais plutôt :

- de préciser les différentes caractéristiques de ces outils de mise en œuvre des recommandations alimentaires chez la vache laitière, en présentant ceux concernant les recommandations françaises ;

- de mettre en évidence les modifications apportées dans les démarches de rationnement, et leur prise en compte dans les outils ;

- de proposer des pistes d'amélioration pour les outils existants.

\section{1 / Qu'est ce qu'un outil de mise en œuvre des recommandations}

Il faut bien différencier les recommandations des outils qui permettent de les mettre en œuvre.

Les recommandations alimentaires peuvent être définies comme une série de règles applicables dans des conditions bien précises (zone de validité), permettant d'atteindre un objectif zootechnique défini par l'utilisateur, à partir de la détermination des apports alimentaires d'une part et de la connaissance des besoins correspondants des animaux d'autre part. Elles s'appuient sur des modèles permettant d'établir des relations entre les différentes fonctions biologiques entre elles et avec les caractéristiques des animaux. Elles font l'objet d'ouvrages décrivant leur fondement scientifique, leur précision et leurs limites (INRA 1988, NRC 1988). Elles peuvent être mises en œuvre à différents niveaux et de différentes manières selon les objectifs de rationnement que l'on se donne (travail à l'échelle du trou-
Figure 1. Schéma d'un outil d'aide àu rationnement.

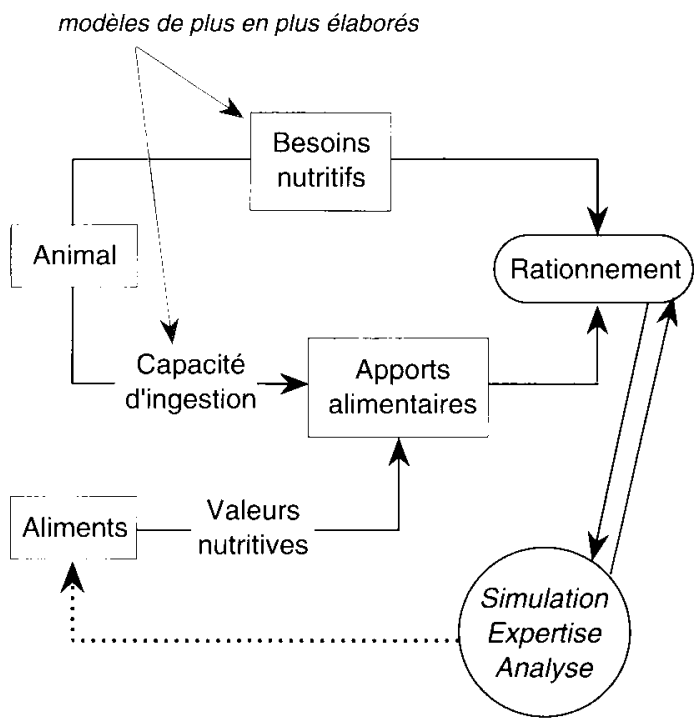

peau, de lots d'animaux, d'individus ; connaissance précise des quantités ingérées de fourrages ou uniquement des apports de concentrés nécessaires ...), et les moyens dont on dispose (voir exemples plus loin).

Les outils de mise en ouvre des recommandations doivent permettre leur application de manière aisée et adaptée aux objectifs de travail. Ils gèrent les relations entre un système de calcul des apports alimentaires (nécessitant la connaissance ou la prévision de la valeur alimentaire des aliments) et un système de détermination des besoins des animaux, en fonction de contraintes techniques et/ou économiques (figure 1). Ils doivent permettre des allers-retours entre ces systèmes et des simulations interactives avec le décideur. La difficulté des outils de rationnement pour bovins

Tableau 1. Mise en œuvre du rationnement de la vache laitière en début de lactation.

1 / Calcul des besoins et des apports nécessaires pour chaque stade de lactation, en fonction des objectifs choisis.

2 / Respect des règles d'application de ces calculs.

Par exemple, limitation du pourcentage de concentré à $50 \%$ pour le calcul de la réduction à appliquer aux apports énergétiques (pour tenir compte des phénomènes d'interactions digestives et métaboliques). L'équation servant à élaborer cette réduction n'a en effet pas été validée pour des proportions supérieures de concentré dans la ration.

\section{3 / Calcul des quantités d'aliments concentrés nécessaires,}

et adaptation de ces quantités pour éviter les variations trop importantes d'une semaine à l'autre et permettre une distribution pratique de l'apport de certains aliments (cas fréquent lorsque l'on utilise un concentré riche en azote, qui peut pratiquement rester fixe au cours des premières semaines de lactation).

\section{4 / Description graphique}

de l'évolution des quantités ingérées, de la production laitière et des bilans nutritifs. Mise en évidence des périodes critiques (déficit énergétique maximal par exemple). Comparaisons des différentes solutions possibles au plan biologique.

\section{5 / Analyse de la ou des solutions.}

Valeur hygiénique, coût. Conséquences d'une variation de l'état corporel au vêlage sur la stratégie d'alimentation. Conséquences d'une modification de cette stratégie par rapport à l'objectif initial sur les performances à court et à moyen terme. 
tient en partie à la grande diversité de situations possibles, liée à la fois à la variété des aliments disponibles (fourrages, concentrés, sous-produits) qui rend difficile l'appréciation de leur valeur alimentaire (ingestibilité principalement), et à la diversité des animaux et de leurs caractéristiques. Ils doivent être adaptés au public visé et au niveau d'approche souhaité. Ils se différencient par leur support (écrit ou informatique), mais surtout par la philosophie générale de leur démarche et la manière d'organiser et de simplifier les résultats, même si les recommandations auxquelles ils se réfêrent sont identiques (c'est le cas des différents logiciels français qui appliquent pour la plupart les recommandations INRA).

Alors que l'élaboration des recommandations alimentaires est le résultat du travail d'un petit nombre de concepteurs (INRA, NRC, ARC ...), il existe une multiplicité de concepteurs pour les outils de mise en œuvre du rationnement.

Pour chaque outil, il faut par ailleurs séparer : 1 ) les calculs proprement dits (besoins des animaux, apports de la ration), 2) les règles d'utilisation de ces résultats (zone de validité des modèles), 3) la mise en œuvre pratique de ces résultats (dans le temps et dans l'espace), 4) la représentation graphique de ces résultats et 5) la présence d'aide à l'interprétation (analyse, expertise). C'est essentiellement sur les points 3,4 et 5 (et dans une moindre mesure sur le point 2) qu'il apparaîtra des différences. On peut illustrer ces différents points à partir de l'exemple du début de la lactation chez la vache laitière (tableau 1). Dans cet exemple, le premier point (calcul des apports) n'est encore qu'incomplètement modélisé en raison essentiellement des lacunes subsistant sur la prévision de la capacité d'ingestion au cours de cette période. L'analyse finale reste par ailleurs à développer (par exemple adaptation du rationnement en fonction de l'état corporel des vaches au tarissement ou au vêlage).

En fait, un outil de mise en œuvre des recommandations doit non seulement se référer à l'ensemble des données comprises dans ces recommandations (et pas uniquement à certaines d'entre elles, ou à certaines de ses zones de validité), mais aussi prendre en compte leur application pratique. Ce dernier point n'a en effet pas été une contrainte majeure dans l'élaboration des différents systèmes qui constituent ces recommandations, ni dans la présentation qui en a été faite dans les ouvrages de référence.

A cela s'ajoute, en particulier pour les logiciels informatiques, les caractéristiques de facilité d'emploi, de rapidité, d'ergonomie...

\section{2 / Les différents outils existants}

Différents types d'outils d'application des recommandations alimentaires sont disponibles (tableau 2). Les outils les plus clas- siques sont les tables, tableaux ou abaques, concernant les aliments (tables de la valeur alimentaire, intégrant les règles de calcul de cette valeur) ou les animaux (besoins alimentaires selon leurs caractéristiques), regroupés par exemple dans les Tables de l'alimentation des bovins ovins et caprins (INRA 1988). On peut les considérer comme les outils de première génération. Ils peuvent être plus ou moins complets : les tables de la valeur nutritive des aliments regroupent ainsi, selon les pays, des nombres variables d'aliments et de caractéristiques, déterminées de manière plus ou moins fiable (cas de la dégradabilité de l'azote par exemple). Ils n'incluent pas ou peu de démarche théorique ou pratique de rationnement. Ils servent de base à une élaboration manuelle du rationnement et nécessitent une mise en forme (calcul) des résultats obtenus.

Parallèlement, il existe des outils d'application pratiques des recommandations, sous forme écrite. C'est le cas des ouvrages généraux de pratique de l'alimentation des bovins (ITEB-EDE 1989) ou de ceux traitant de certaines rations particulières (Chenais et al 1990). Ils présentent sous forme appliquée, précalculée et de manière simplifiée les résultats obtenus à partir des recommandations afin de proposer des solutions pratiques conduisant à des rations types directement utilisables sur le terrain, accompagnées de repères et de conseils pratiques permettant de juger de la qualité du rationnement. Ces rations intègrent souvent une variation par rapport au normes biologiques, de manière à assurer un optimum technico-économique. Elles s'appuient sur des notions déjà connues (lait permis par la ration de base, rythme de distribution du concentré, concentré correcteur...).

Enfin, il existe un certain nombre de logiciels intégrant le rationnement des animaux. Parmi l'ensemble des logiciels disponibles en France concernant la gestion technico-économique des troupeaux laitiers (une vingtaine répertoriée par l'ACTA (1991) et le CXP (1991)), si la plupart intègrent la notion de rationnement, peu en font leur objectif princi-

Tableau 2. Les différents types d'outils d'aide au rationnement.

\begin{tabular}{|c|c|c|}
\hline $\begin{array}{c}\text { Type } \\
\text { d'outil }\end{array}$ & $\begin{array}{c}\text { Rôle } \\
\text { de l'outil }\end{array}$ & $\begin{array}{c}\text { Rôle } \\
\text { de l'utilisateur }\end{array}$ \\
\hline Tables & Valeurs & Calculs de solutions \\
Abaques & (besoins, & Adaptation \\
Tableaux & valeur nutritive) & Expertise \\
Ouvrages & Calcul de solutions & Adaptation \\
Logiciels & Pré-adaptation & Expertise \\
& Simplification & Simulation \\
& Pré-expertise & Expertise \\
Logiciels & Calculs & \\
& Adaptation & Cimulation \\
& Calculs & Contre-expertise \\
Logiciels & Adaptation & \\
& Expertise biologique & \\
& Expertise économique & \\
& &
\end{tabular}


pal, et certains ne réalisent pas en fait de calcul de ration, mais proposent une analyse d'une ration existante. En fait la majeure partie des logiciels français et étrangers s'attachent d'abord à donner rapidement une image précise de la situation de l'exploitation ou du troupeau, en particulier sur des aspects événementiels (santé, reproduction), et/ou à gérer des évolutions de production laitière (quotas). Certains proposent une expertise de la situation observée mais rarement en matière d'alimentation.

Les recommandations étrangères ont pour la plupart été mises sur support informatique. Dans certains cas, cet outil n'est qu'un moyen de formaliser une suite d'équations, et ne met pas en ouvre de solutions pratiques (cas du NRC). Il existe cependant des logiciels à finalité économique se basant sur ces recommandations et traitant uniquement ou essentiellement de rationnement (Jones et al 1980 , Hulme et al 1986, Weaver et al 1988).

\section{3 / INRAtion : un outil d'application des recommandations françaises}

Lors de la présentation des nouvelles recommandations pour les vaches laitières en janvier 1988, différentes méthodes de calcul du rationnement avaient été proposées, permettant d'illustrer l'influence de l'objectif visé sur les outils mis en œuvre (Faverdin et al 1987). Ces différentes méthodes s'appuyaient sur les mêmes bases, mais ne permettaient pas le même niveau de précision. Les 3 premières ne représentaient d'autre part qu'un cas d'école, sans fournir de mise en œuvre pratique, ce que fait au contraire l'ouvrage "Pratique de l'alimentation des bovins" (ITEB-EDE 1989). La 4ème correspondait au logiciel INRAtion. Dans sa première version, ce logiciel s'attachait surtout à traduire de manière fiable et rapide les nouveaux systèmes de rationnement. Les versions ultérieures ont permis d'enrichir de manière importante l'approche pratique du rationnement.

INRAtion est un logiciel de calcul et d'analyse du rationnement multi-espèces, prenant en compte l'ensemble des connaissances exposées aux cours du 17ème Grenier de Theix en janvier 1988, ainsi que celles disponibles depuis. Il les organise de manière 1) à proposer à l'utilisateur une ou des solutions théoriques validées dans une large gamme de situations, 2) à permettre leur analyse, et 3) à préparer leur application pratique ultérieure. Ce n'est donc pas un logiciel destiné à fournir directement une ration type à un éleveur selon ses contraintes et ses pratiques, même si ses modules de calculs peuvent être (et sont déjà) utilisés dans cet objectif, en étant intégrés à des outils plus généraux. Il se différencie en cela des logiciels de gestion technico-économique des troupeaux développés par ailleurs
(ACTA 1991). Il est d'ailleurs en priorité destiné aux agents du Développement, de l'Enseignement, de la Profession et de la Recherche, plutôt qu'aux éleveurs eux-mêmes.

INRAtion met en œuvre l'ensemble des modèles de calculs des besoins des animaux utilisés par les recommandations dans leur intégrité, et une démarche de calcul de la ration identique et hiérarchisée, quelles que soient la situation et l'espèce considérée. Cette démarche, lourde en calculs, est totalement transparente pour l'utilisateur et aboutit à la fourniture d'un certain nombre de solutions théoriquement satisfaisantes et validées, compte-tenu des objectifs affichés et des valeurs des aliments proposées. Il s'agit là des 2 premières étapes telles qu'elles ont été définies précédemment. A ce niveau, des notions pratiques (telle que celle de correcteur azoté) ne sont pas intégrées dans la démarche de calcul. Elles ne sont en effet pas universelles. Elles seront éventuellement déduites des résultats présentés dans la 3ème étape (mise en œuvre pratique). Par contre, le mode de distribution des aliments (ration complète ou séparée) est intégré dès cette étape, parce qu'il modifie le comportement biologique des animaux (capacité d'ingestion) et nécessite l'utilisation d'un modèle de calcul particulier. A partir de là, un certain nombre de modules d'aide à la décision sont disponibles (coût des différentes solutions, conséquences d'une modification des apports par rapport à la solution de référence...) et permettent de choisir une solution privilégiée. Celle ci peut être alors appliquée à l'ensemble du troupeau, et simplifiée en fonction de contraintes pratiques, tout en restant dans une zone de validité biologique. Enfin les conséquences du choix d'une solution donnée à l'échelle du troupeau et sur une période de temps donnée peuvent être calculées (quantités d'aliments nécessaires). Le tableau 3 illustre cette démarche à partir de l'exemple de l'étude d'une ration complète.

L'utilisation du logiciel INRAtion nécessite la définition précise de l'objectif que l'on se donne. Il permet alors de résoudre des problèmes différents, d'ordre 1) descriptif : validité d'une ration existante. Dans ce cas, le logiciel donnera toujours l'état des lieux, même s'il est mauvais. Il joue le rôle d'outil de diagnostic. 2) prévisionnel : quelles quantités d'aliments seront ingérées ou sont à distribuer, selon les objectifs fixés. Dans ce cas, le logiciel propose une ou plusieurs solutions ; mais il peut aussi n'en proposer aucune, en précisant les raisons. 3) pédagogique : par exemple, illustration de la notion de substitution, des interactions fourrages/concentrés, de l'intérêt et des limites de la ration complète par rapport à la ration séparée... 4) scientifique : par l'aide qu'il peut apporter à l'élaboration d'un protocole expérimental (prévision de l'écart d'apport énergétique entre 2 lots par exemple). 
Tableau 3. Démarche d'application d'INRAtion au rationnement d'un troupeau de vaches laitières recevant une ration complète.

\section{Objectif: Prévoir les quantités ou les proportions de différents aliments dans la ration} pour un troupeau de vaches dont la production varie de 25 à $45 \mathrm{~kg} / \mathrm{j}$.

\begin{tabular}{llc}
\cline { 2 - 3 } Aliments disponibles et & \multicolumn{1}{c}{ Fourrages } & \% dans la ration \\
\cline { 2 - 3 } contraintes éventuelles : & ensilage herbe & 10 \\
& ensilage maìs & à déterminer \\
& paille & 5 \\
pulpes & 10
\end{tabular}

\begin{tabular}{ll} 
Concentrés & \% dans la ration \\
\hline orge & à déterminer \\
soja & à déterminer \\
urée & à déterminer
\end{tabular}

Démarche de travail :

Quelle(s) solution(s) théorique(s) pour le niveau de production moyen $(35 \mathrm{~kg} / \mathrm{j})$ ?

Deux solutions sont possibles :

Objectifs :

Energie : $100 \%$

Azote : $100 \%$

Caractéristiques des animaux

Poids : $700 \mathrm{~kg}$

TB : $40 \mathrm{~g} / \mathrm{kg}$

TP : $32 \mathrm{~g} / \mathrm{kg}$

\begin{tabular}{|c|c|c|}
\hline Ration complète & $\begin{array}{r}\text { Proporti } \\
\text { alimen } \\
\text { solution } 1\end{array}$ & $\begin{array}{l}\text { Ions des } \\
\text { ts (\%) } \\
\text { solution } 2\end{array}$ \\
\hline ensilage herbe & 10,0 & 10,0 \\
\hline ensilage maïs & 36,1 & 36,1 \\
\hline paille & 5,0 & 5,0 \\
\hline pulpes & 10,0 & 10,0 \\
\hline orge & 31,6 & 29,1 \\
\hline soja 48 & 7,4 & 9,8 \\
\hline urée & 0,5 & \\
\hline$\%$ UFL & 101 & 101 \\
\hline$\%$ PDIN & 100 & 100 \\
\hline$\%$ PDIE & 100 & 104 \\
\hline
\end{tabular}

Elles diffèrent par la présence d'urée et les parts respectives d'orge et de soja dans la ration.

Choix d'une solution (la seconde).

Conséquences zootechniques de son application en conservant les proportions à l'échelle du troupeau :

\begin{tabular}{|c|c|c|c|c|c|}
\hline Lait $(\mathrm{kg} / \mathrm{j})$ & 25 & 30 & 35 & 40 & 45 \\
\hline ens.herbe & 2,0 & 2,2 & 2,3 & 2,4 & 2,5 \\
\hline ens.maïs & 7,2 & 7,8 & 8,3 & 8,7 & $9, \uparrow$ \\
\hline paille & 1,0 & 1,1 & 1,2 & 1,2 & 1,3 \\
\hline pulpes & 2,0 & 2,2 & 2,3 & 2,4 & 2,5 \\
\hline orge & 5,84 & 6,31 & 6,70 & 7,04 & 7,32 \\
\hline soja 48 & 1,96 & 2,12 & 2,25 & 2,37 & 2,46 \\
\hline ration & 20,1 & 21,7 & 23,0 & 24,2 & 25,1 \\
\hline \multicolumn{6}{|l|}{ Bilans } \\
\hline UFL & 1,8 & 1,1 & 0,0 & $-1,2$ & $-2,6$ \\
\hline PDIN & 207 & 113 & -1 & -135 & -285 \\
\hline PDIE & 281 & 193 & 84 & -46 & -192 \\
\hline
\end{tabular}

Cette ration conduit à sous-alimenter fortement en énergie et en azote les animaux les plus forts producteurs.
Amélioration de la ration :

Constitution d'une ration semi-complète pour les animaux les plus forts producteurs.

Composition de la ration

ensilage herbe : $10 \%$

ensilage maïs : $35 \%$

paille : $5 \%$

pulpes $\quad: 10 \%$

orge $: 30 \%$

soja $48 \quad: 10 \%$

- Module troupeau-

\begin{tabular}{llllll} 
Lait $(\mathrm{kg} / \mathrm{j})$ & 25 & 30 & 35 & 40 & 45 \\
\hline ens.herbe & 2,0 & 2,2 & 2,3 & 2,4 & 2,5
\end{tabular}

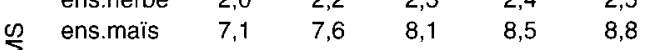

$\begin{array}{llllll}\sum_{0} \text { paille } & 1,0 & 1,1 & 1,2 & 1,2 & 1,3\end{array}$

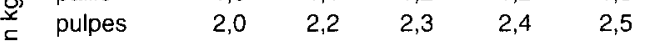

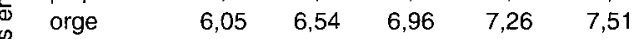

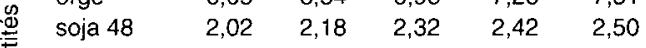

ration $20,2 \quad 21,8 \quad 23,2 \quad 24,2 \quad 25,0$

$\begin{array}{lll}\text { soja } 48 & 0,44 & 0,88\end{array}$

\begin{tabular}{lccccc} 
Bilans & & & & & \\
UFL & 1,9 & 1,2 & 0,2 & $-0,9$ & $-2,2$ \\
PDIN & 233 & 145 & 36 & 53 & 56 \\
PDIE & 304 & 222 & 117 & 86 & 40 \\
\hline
\end{tabular}

L'ajout de 0,5 à $1 \mathrm{~kg}$ de tourteau de soja à la ration des vaches produisant plus de $40 \mathrm{~kg} / \mathrm{j}$ permet d'éviter le déficit azoté et de réduire le déficit énergétique. Celui-ci reste acceptable compte tenu. du niveau de production et du stade de lactation de ces vaches (encore au début de lactation).

Simplification de la ration :

Les pourcentages des différents aliments (ou leurs quantités peuvent être arrondis sans conséquence sur les bilans nutritifs (cf plus haut).

Le calcul du coût de cette ration et des quantités d'aliments nécessaires à sa mise en oeuvre à l'échelle de plusieurs semaines peut ensuite être réalisé. 
Il présente par ailleurs un certain nombre de limites :

1) liées à l'état des connaissances, ou à l'absence de modèles validés permettant leur application pratique : absence d'appréciation de la valeur hygiénique des rations, non prise en compte de l'état corporel dans le rationnement des vaches laitières, pourcentage limité de concentré dans la ration, difficultés de réalisation de certaines rations avec certains aliments peu connus, en particulier certains sous-produits.

2) liées aux objectifs initiaux : certaines catégories d'animaux (vaches taries) ne sont pas prise en compte ; certains aspects du rationnement sont peu développés (cas du rationnement minéral); certaines rations ne sont abordées que de manière théorique : c'est particulièrement le cas du rationnement au pâturage, qui ne prend pas en compte les aspects propres au pâturage (besoins nutritifs supplémentaires, facteurs de variations des quantités ingérées), contrairement à des outils étrangers (CSIRO 1990) : il s'agit en fait de calcul de ration avec de l'herbe coupée.

3) liées aux priorités définies dans sa conception : il n'y a pas d'optimisation économique conjointe à l'optimisation nutritionnelle. La prévision de la valeur alimentaire des aliments n'est pas intégrée. L'expertise des résultats n'est pas organisée. Le graphisme est encore peu développé.

\section{Conclusion}

La gamme des outils disponibles pour réaliser le rationnement des vaches laitières, ainsi que leur efficacité, s'est considérablement accrue depuis 1978. Parallèlement, la gamme des situations d'application s'est aussi élargie, d'un point de vue technique (niveau de production des animaux, variété des aliments) et économique (contraintes extérieures liées à la qualité du lait et/ou à son contingentement). Il est donc nécessaire, pour profiter utilement du confort d'utilisation de ces nouveaux outils, d'une part de connaître suffisamment les principes et les limites des systèmes qu'ils mettent en œuvre, d'autre part de préciser les exigences et les objectifs de l'utilisateur. Les facilités permises par l'informatique ne doivent s'appliquer qu'à la mise en œuvre et à la présentation des calculs. Elle ne remplace pas la réflexion nécessaire (et certainement plus complexe que par le passé pour les raisons précisées précédemment) à leur préparation et à leur application. En cela, un outil comme INRAtion se conçoit comme un outil de référence, au même titre que le Livre Rouge ou le Bulletin de Theix $\mathrm{n}^{\circ} 70$ sont des ouvrages de référence, permettant la mise à disposition rapide et organisée des connaissances, mais non utilisable indépendamment de ces ouvrages.

A l'avenir, on peut penser qu'à coté d'une amélioration ergonomique, l'essentięl de l'amélioration scientifique des outils de rationnement sera lié à la prise en compte des connais- sances nouvelles, et surtout à l'intégration de l'expertise des résultats proposés (tableau 2) (Spahr et al 1988, Whittaker et al 1989 , Hogeveen et al 1991).

\section{Références bibliographiques}

ACTA, 1991. Progiciels agricoles pour microordinateurs. Catalogue 1991. 271 p. ACTA, 149 rue de Bercy, Paris.

AFRC, 1990. AFRC technical committee on responses to nutrients, report number 5 , nutritive requirements of ruminant animals : energy. Nutr. Abst. Rev. (B), 60, 729-804.

Chenais F., Augeard P., Bazin S., Martial J.P., Masson D., 1990. Les rations complètes à base d'ensilage de maïs. Atouts et mise en oeuvre. Collection "le point sur". 48 p. ITEB, 149 rue de Bercy, Paris.

CSIRO, 1990. Feeding standards for Australian Livestock Ruminants. CSIRO Publications. Victoria 30002. Australia

CXP 1991. Farmsoft 91. Catalogue international des logiciels agricoles. 175p.

Faverdin P., Hoden A., Coulon J.B., 1987. Recommandations alimentaires pour les vaches laitières. Bull. Tech. CRZV Theix, INRA, 70, 133152.

Hogeveen H., Noordhuizen-Stassen E.N., Schreinemarkers J.F., Brand A., 1991. Development of an integrated knowledge-based system for management support on dairy farms. J. Dairy Sci., $74,4377-4384$.

Hulme D.J., Kellaway R.C., Booth P.J., 1986. The Camdairy model for formulating and analysing dairy cow rations. Agric. Syst., 22, 81-108.

INRA 1988. Alimentation des bovins, ovins et caprins. ed R. Jarrige. 471 p. INRA, 147 rue de l'Université, Paris.

ITEB, EDE, 1989. Pratique de l'alimentation des bovins. 186 p. ITEB, 149 rue de Bercy, Paris.

NRC, 1988. Nutrient requirements of dairy cattle. Sicth revised edition. National Academy Press, Washington D.C. 157 p.

Spahr S.L, Jones R., Dill D.E., 1988. Expert systems-their use in dairy herd management. J. Dairy Sci., 71, 879-885.

Weaver L.D., Olivas M.A., Galland J.C., 1988. Identifying features, performance, and limitations of dairy ration formulating software : a comparison of three ration formulation programs. J. Dairy Sci., 71, 1104-1115.

Whittaker A.D., Tomaszewski M.A., Taylor F.J., Fourdraine R., Van Overveld C.J., Schepers R.G., 1989. Dairy herd nutritional analysis using knowledge systems techniques. Agric. Syst., 31, 8396. 


\section{Summary}

A study of some feeding systems for ruminants : different methods of application.

Ruminant feed systems and feed recommendations which ensue have been considerably modified during these last years, both in France and abroad. In parallel, the application methods for these recommendations have diversified. In France these methods have been mainly recommended by I.N.R.A. (National Institute for Agronomic Research). It is thus important to differentiate feed recommendations and the methods which enable these recommendations to be carried out. Among these methods there are tables (which provide for example feed value), books which pre- sent in simplified and pro-calculated form, practical solutions which are directly usable by farmers, as well as computer programmes such as INRAtion. In this study the different characteristics of methods for putting feed recommendations into practice are illustrated using the example of rationing in dairy cattle. The interest and future development of computers are discussed.

COULON J.B., AGABRIEL J., 1992. Réflexions sur les outils de mise en oeuvre des recommandations alimentaires. Application aux vaches laitières dans les conditions françaises. INRA Prod. Anim., 5 (5) 347-353. 